\title{
A methodology to improve a public transport system
}

\author{
G. Salvo, L. Caruso \& N. Santoro \\ Department of Energy, Transportation Research Group, \\ University of Palermo, Italy
}

\begin{abstract}
The quality of pedestrian routes of access to a public transport system (PTS) is an important aspect for the choice of transport mode, which can be evaluated using some indicators such as accessibility, comfort, frequency, etc. The policies for urban sustainable mobility must reflect the design and management of mass transit systems, but also the judgment of users in terms of quality and perception of PTS. The purpose is to reassess slow mobility encouraging the intermodal mobility between pedestrian and public transport. Walking can be considered as an alternative mode of urban transport and a moment of a break from the chaos of modern life. Its role should be considered as an important element for a new model of structuring the city. Using geomatics a methodology for the assessment of accessibility to the PTS has been developed, that takes into account the quality of a pedestrian path to reach the bus stop. The distance between bus-stops, human energy expended to reach the stops of the PTS, etc, are some elements analyzed in this study. The qualitative and quantitative data of the pedestrian network access have been managed in a Geographic Information System (GIS), proving a valuable tool that can handle a large amount of spatial and heterogeneous data. The results can be of support both at the planning/strategic level that also helps the users, if inserted in the context of a Web-GIS, making the integration between pedestrian mobility and PTS competitive.

Keywords: public transport system, pedestrian mobility, geomatics.
\end{abstract}




\section{Introduction}

In recent decades, the increase of urban mobility demand has been met by the use of private cars, which has determined a freedom and comfort in the movements; but it has created also problems of traffic congestion.

The results are consequences on safety, urban life, quality of services and acoustic/air pollution.

New road and/or their adaptation to the increased traffic flows are long-term interventions that are often inefficient for the congestion that occurs every day.

In fact, the improving of road infrastructures determines an increasing of the accessibility, but also these interventions increase the traffic generated causing a fall-off level of service and then a further increase in road traffic.

So the private transport is not functional and unsustainable, especially within urban areas. New interventions are necessary, improving all forms of the sustainable mobility, which promote ecological transports.

Principal problems related to public transports are connected to architectural barriers, which are in many urban areas and inside public transport.

In fact, mobility in the city is very difficult, especially for those people affected by mobility difficulties or sensory impairments.

It should, therefore, improve urban accessibility, that it must be an objective for improving the quality of city life.

Accessibility in urban area contains a complex series of:

- legislative,

- technical measures,

- space-based solutions,

- modes of organization for efficient service.

This is designed to elevate the usability and safety of the built environment and reinforce the comfort.

To improve the accessibility and therefore usability of the built space, all the dangers and discomfort should be eliminated, by stimulating the action of walking.

The distances to be done walking must be limited and protected and waiting areas of public transport facilities should be set up along the route.

Bus stop location has significant importance in both urban and rural trips, such that access to them is attributed the same importance on the perception of safety and comfort during movement.

The correct positioning of the stops is a critical element for the safety of passengers and pedestrians, as to the effectiveness and efficiency of operation of the TPL.

The parameters, generally, considered for the location of the stops are physical, such as the maximum distance and maximum travel distance.

The parameters related to the quality of pedestrian access to the bus stop, their actual length and land use in the neighborhood of the station are not considered [1]. 
The objective of this paper is to propose a method, through the use of georeferencing of information technologies, which can correlate the quality and quantity of the pedestrian network access to the bus stop.

The methodology, proposed in this paper, revaluates the walking, that can be seen not only as a break moment from the chaos of modern life, but also as an alternative mode of urban transport.

Pedestrian mobility and public transports should be considered as important elements for a new model of cities, in relation not only to new parts, but also to old and suburban areas, where streets and squares, created for pedestrians, were abused by vehicular traffic, more intense, fast and heavy.

Urban frame of cities, where is priority the use of private cars, must be redesigned to meet the diverse needs of vulnerable road users, taking into account how they are often forced to use facilities where mobility is unsafe and unpleasant.

\section{Review of scientific literature}

In the scientific literature modal integration between bus and walking public, is presented as an alternative to the private car, because allows to achieve a high level of mobility, without the same negative impact on the environment (externalities).

The literature review has reported several applications on pedestrian safety, sometimes developing quality indicators and models applied to the behavior of pedestrians.

The increase in this mode of transportation determines many benefits for all citizens, both in environmental, but also social and energy due to the reduction of congestion of motor traffic.

The public transport has some critical points, such as:

- Lack of capillarity;

- No intermodality with other transport systems;

- Overcrowding of vehicles;

- Lack of cleanliness and comfort;

- Inadequacy of times;

- Travel times uncertain due to the need to make many stops along the way;

- Poor quality of pedestrian access/egress to BUS.

A correct bus stop a location is, therefore, an aspect to analyse for improved PTS, minimizing the distance that the user must make on walking.

In fact, the spacing of bus stops has a significant impact on the vehicle performance and system of public transport because the distance between the stops also influences the overall time of travel and, consequently, the demand for transport.

Some authors have studied the localization of the stops in a line of urban public transport through a network approach for the representation of pedestrian accessibility, defining a methodology that takes into account the point of view before taking the vehicle, minimizing the movement walk, and performance for the service, with the optimization of the commercial speed [2]. 
The access to the LPT system is therefore influenced by the perception of safety, comfort and pleasantness of the movement on foot, which depends on the quality of the pedestrian path, as important as the distance traveled.

Various studies have permitted significant progress in the development of indices of service to measure the quality of the pedestrian path from / towards the stop [3].

In the Italian legislation there isn't a law protecting the accessibility and safety of pedestrian.

The actual Italian legislation in particular represented by the Highway Code [4] and its Implementing Regulations and their implementation, which provides: "In towns and on the open road, the stops of vehicles (comma 1) situated in correspondence of the areas of intersection, are located, after the area of intersection, at a distance of not less than $20 \mathrm{~m}$. The stop must be anticipated at least $10 \mathrm{~m}$ from the intersection, if the number of lines and the frequency of trips due to accumulation of the means so as to interfere with the operation for the area of intersection".

In the absence of Italian legislation on traffic calming, which protects the pedestrian walkways and around public transport stops, often the problem has been resolved by transposing regulations in some European countries. In fact, some Italian regions have adopted some European Directives on accessibility and security.

In Europe there are many experiences that demonstrate how regeneration road, through a redesign of the space, can make compatible the different functions of the urban street, as movement, walking, resting and also increase safety for all users .

In particular the American regulations have written guides and reports from AASHTO, ITE, ASCE, and FHWA. The ASHTOO recommends locating the stops of the TPL in the immediate vicinity of an intersection.

This is a benefit for those who cross the road, without the need for intermediate crossings, preferring the location after the intersection, especially along the arteries with right of way.

Many cities have a real "Sidewalk Regulation" containing guidelines on the minimum requirements they must meet and possible improvements in order to pursue objectives of safety, liveability and attractiveness of routes.

Highway Capacity Manual [5] analyzes the pedestrian access to public transport line with methods based on the calculation of the level of service (LOS) by performing a functional study based on the relationships that link the descriptive variables of the same level of service vehicle.

Other methods [6] quantifying the perception of safety and comfort of pedestrians that moving in the urban road as a function of physical elements that characterize the sidewalk, taking into account the lateral separation from vehicular traffic and the presence of barriers, not just natural.

Finally, the manual design of cycling facilities prepared by the Netherlands Centre CROW defines the loss of energy of the cyclist as a function of different physical and environmental parameters, taking into account the mass of the cyclist, the bicycle, the slope of the path, the speed reached and the wind speed [7]. 


\section{Case study}

The urban growth of Palermo can be divided into two phases:

- Before the Second World War in which Its development is linear along the axis of Via Oreto, Maqueda and Libertà;

- Today It has a dimension of metropolitan area, characterized by a radial pattern developed to semicircle form, due to the morphology of the area.

This last process has focused on strict guidelines for growth, involving all directions of the semicircle, leaving out the areas where natural barriers have kept the urbanization.

In this paper we analyze Viale Lazio street, affected by this expansion.

This street belongs to the eighth district, which connects the east area of the city with Viale Regione Siciliana (urban freeway). It is an important road that links north and south of the city, extending for a distance of about $1 \mathrm{~km}$ with a road width of about 12 meters (fig. 1).

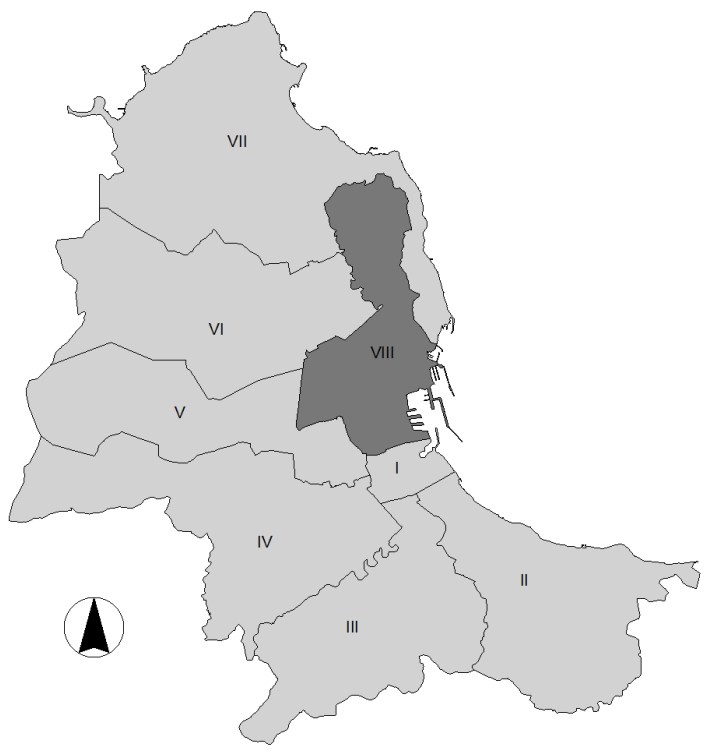

Figure 1: Urban district of Palermo.

There are various commercial activities and public offices, such as the Anagraphical and Demographics Services, etc.

\section{Methodology}

This study proposes a methodology to improve the walking/PTS comfort, in relation to the distance between the bus stops and points of interest that are present in the area. 
In particular we analyze the energy expended to reach the stop of the PTS and safety-pleasantness of pedestrian travel route.

In fact the acclivity, the total mass transported, the microclimatic conditions are just a few elements that characterize the energy used [7] to walk those distances.

The expression to determine the energy spent during walking, determined on the basis of similar studies, is the following:

$$
P=\left(0.0981 \cdot i \cdot m+0.0721 \cdot m+0.374 \cdot v^{2}\right) \cdot v
$$

$\mathrm{P}=$ power required to keep the constant speed $\mathrm{v}$ (watt)

$\mathrm{i}=$ the slope of the path $(\%)$

$\mathrm{m}=$ total mass (pedestrian and a hypothetic increased load carried) $(\mathrm{kg})$

$\mathrm{v}=$ desired speed $(\mathrm{m} / \mathrm{s})$

The speed " $v$ " of pedestrian standards (old 40-50 years) is constant and equal to $1.2 \mathrm{~m} / \mathrm{s}$ [5], while the mass is assumed equal to $70 \mathrm{~kg}$ and $1.5 \mathrm{~kg}$ load transported.

Index of difficulty walking (In.Di.Pe) was also defined as the ratio "Ls", corresponding to the work produced by the maximum sustainable effort to overcome a path length of $300 \mathrm{~m}$ (average distance to be traveled to reach bus stop) and "L" (the work necessary to walking an arc of length "l" and slope "i"):

$$
\begin{gathered}
\text { In.Di.Pe }=\frac{L}{L_{S}} \\
L=P \cdot 1 \quad(\mathrm{watt} \cdot \mathrm{m}) \\
L s=P s \cdot 300 \quad(\mathrm{watt} \cdot \mathrm{m})
\end{gathered}
$$

In the literature it is clear that for the type of user in question, to overcome a path length of $300 \mathrm{~m}$, it is necessary to provide a power Ps equal to 14 watts approximately in plain with asphalt [7]. This index can to assess the degree of difficulty that every single arc requires for its overcoming, in relation to the physical capacity and performance of the user.

In particular for values lower than unity the user can walk the path without interruptions, using his available power.

For values equal to unity he is in the limit condition where full power supplied is spent to cover the arch, and finally to higher unit values do not exceed the section in question for which the pedestrian is forced into a recovery physical.

For each bus stop is determined the area on which there are more arcs " $\mathrm{j}$,, associating to each arc a pedestrian speed "v", and, for each of them the "In.Di. Pe.-j ".

The calculation of the index to associate the entire route, is not the result of algebraic summation of the individual indices "In.Di.Pe.-j", because the pedestrian is affected by the efforts to go to the arcs. After the overcoming of the 
first arc, the quantity of spent residual power "Pr" is evaluated.

This process is repeated for all of the route selected and it is arrives, so, to determine the effort that the user must bear in order to make the shift.

Index obtained is characterized by the degree of physical difficulty to which the user is subjected before it can to access the TPL and it was divided into three classes:

- difficulty acceptable, if the value of the index that is obtained it is $<1$;

- $\quad$ difficulty sustainable, if the value is $=1$;

- difficulty unsustainable, if I $>1$.

GPS located along the section of Viale Lazio the position of the various bus stops in both directions is found. The waypoints were entered into a Geographic Information System (GIS) and they have permitted to divide the study area into different sections of the macro variable size corresponding to the area of pavement between two bus-stops later, and then calculating the relative distance (fig. 2).

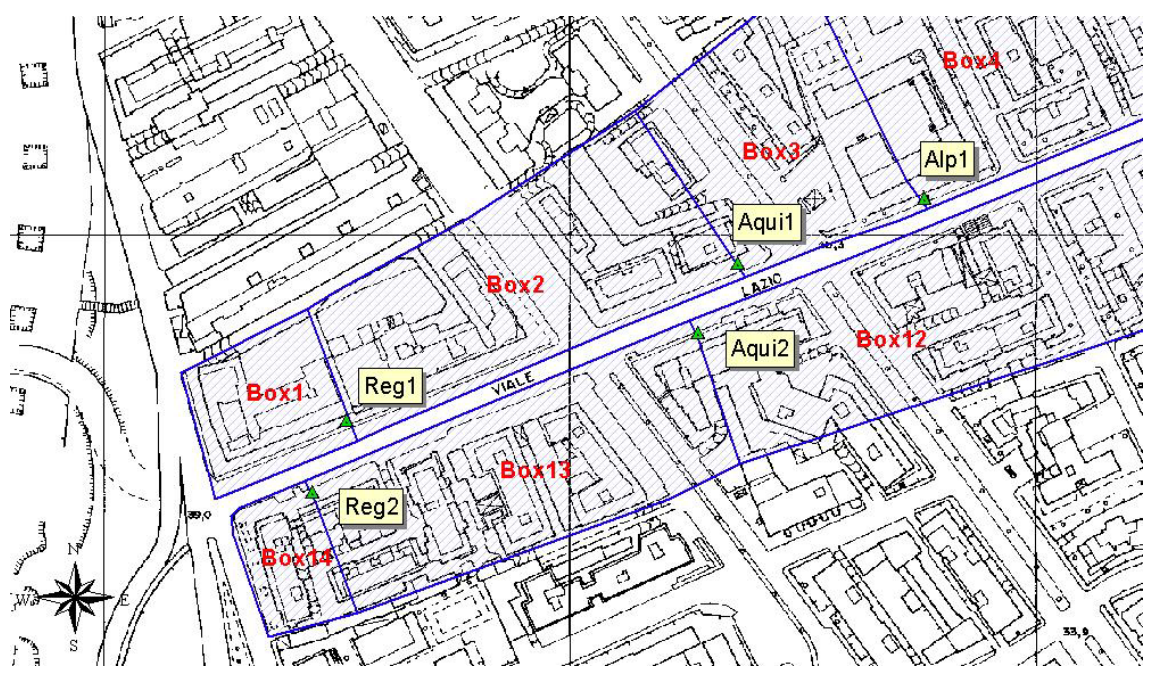

Figure 2: The box area between two bus-stops.

The In.Di.Pe was calculated for each box obtaining, thus, the GIS thematic map of In.Di.Pe.. A database was also collected using information about other attractive elements related to the area, such as public offices, various shops, education centers, etc.

They are included in generators of mobility shapefile, as polygon entities.

The ID and a field representative of the classification of mobility generated are inserted into the table of attributes, with the following categories:

- low interest (activities of local interest or neighborhood);

- high interest (activities of great attractiveness, as municipal offices, shopping centers, community services, etc.) (Fig. 3). 


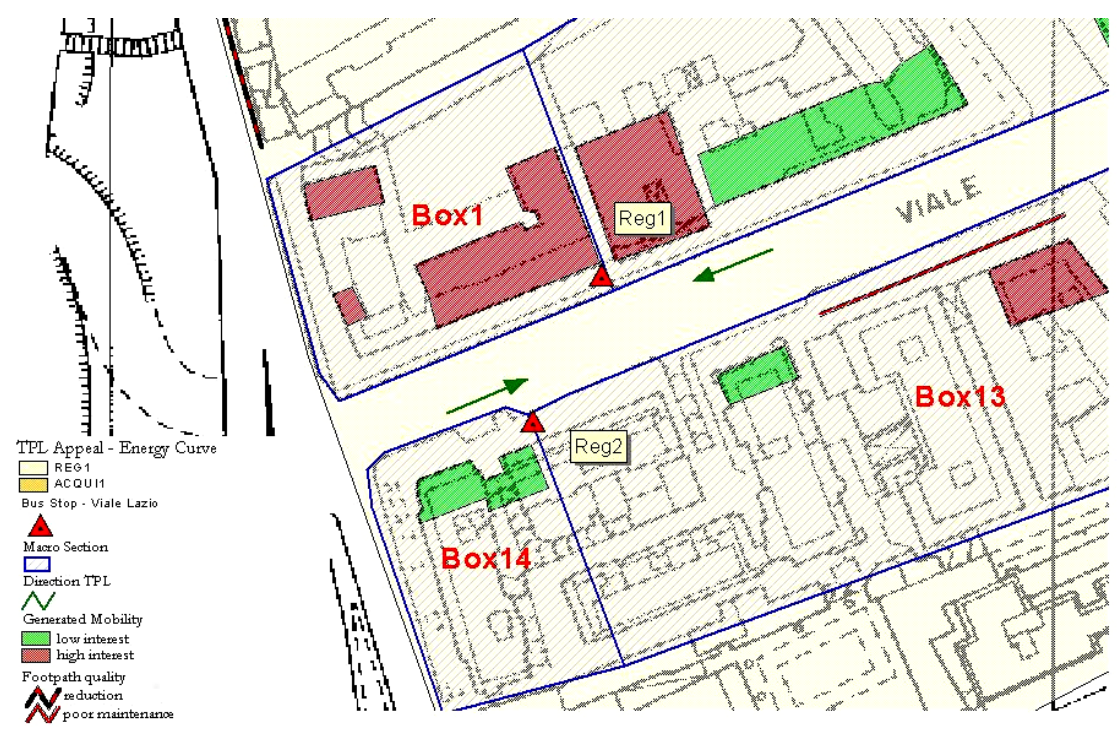

Figure 3: The attractiveness areas of the PTS.

This elaboration overlayed to the In.Di.Pe. map can highlight the points of interest in the examined area, the attractiveness of the PTS and the zone where the consumer energy by pedestrian is acceptable to reach the nearest bus stop (Fig. 4).

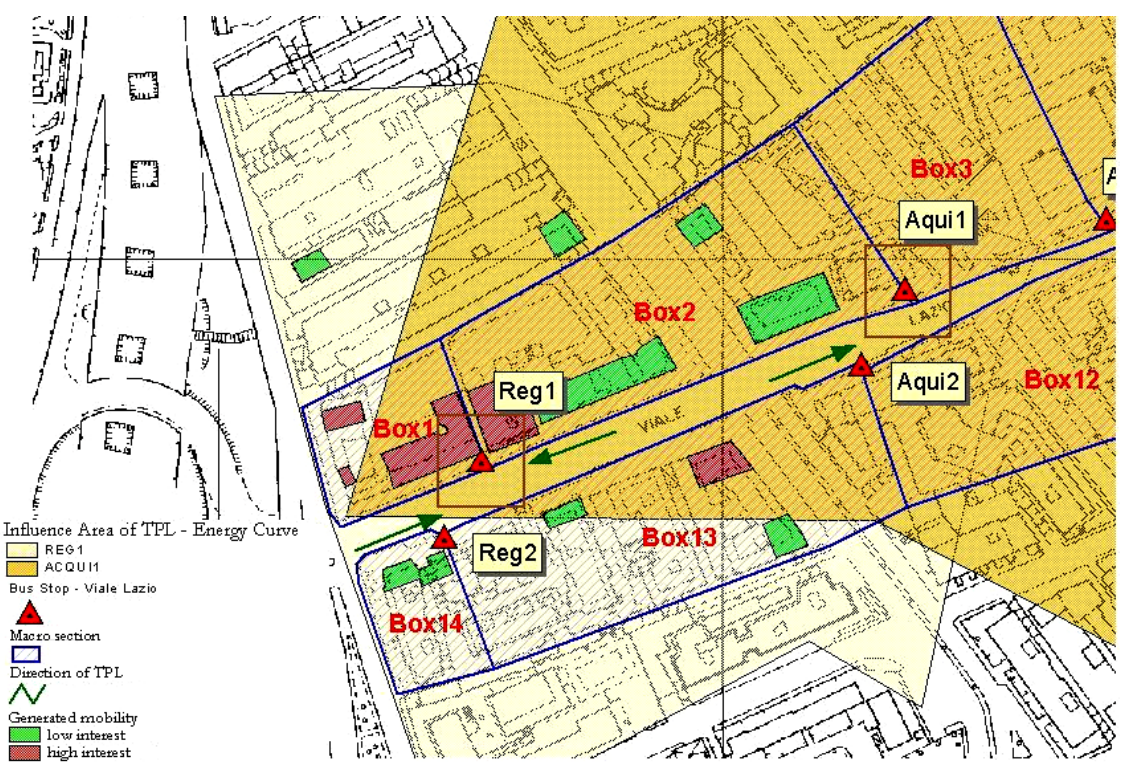

Figure 4: $\quad$ Energy curves. 
The quality of pedestrian shape is obtained from the intersection of the shapefile on the sidewalk width with the state of pavement wear. The target is to assess the quality of pedestrian access - egress to public transport.

HCM defines an acceptable width of the sidewalk for pedestrian traffic, at least $0.75 \mathrm{~m}$, so for each box were highlighted its critical situations.

Finally by adding of the processing can be obtained to evaluate the user accessibility to public transport, highlighting the potential of this modal integration, defining all possible critical situations.

Information for each macro section are inserted into tabs synoptic, so as to be a valuable tool to support and immediate for those concerned with the use of mass public transit service.

\section{Results and conclusions}

This analysis shows that the axis of Viale Lazio is characterized by a good accessibility to TPL, although in some box the wear state or the width of the pavement, make unattractive this mode of transport.

Bus stop locations and human energy to reach them, according also to the slope of this infrastructure road, are acceptable, demonstrating an efficient localization in relation to places of interest.

The purpose of this study is to encourage modal integration between walking and public transport, not only for the sustainable mobility but also because there are obvious advantages for the physical health.

The developed GIS is a valuable tool that can handle a large amount of heterogeneous geo-referenced spatial data, processing them to obtain alphanumeric and visually summary information. In this way these data processed can be used to planning at the strategic level but also by users through a web-GIS.

\section{References}

[1] Salvo, Santoro, Accessibilità pedonale ad una rete di trasporto pubblico, In International Conference Living and Walking in cities, Brescia, 2011.

[2] Ignaccolo, Inturri, Caprì, Localizzazione delle fermate di una linea di trasporto pubblico urbano mediante un approccio di rete per la rappresentazione dell'accessibilità pedonale, SIDT - XIII SEMINARIO - Il Trasporto Pubblico Locale - Pisa 2005.

[3] Salvo, Santoro, Un indice di qualità per l'accessibilità al trasporto pubblico, in Convegno Sistema Gomma nel TPL - ASSTRA, Roma, 2011.

[4] Italian Highway Code, Dlgs. 30 Aprile 1992 n.285.

[5] HCM. Highway Capacity Manual, (eds) TRB of the National Academes, 2010.

[6] Landis, Bruce W., Venkat R. Vattikuti, Russell M. Ottenberg, Douglas S. McLeod, Martin G., Modeling the Roadside Walking Environment: A Pedestrian Level of Service, Transportation Research Record 1773. Transportation Research Board, Washington, DC, 2001.

[7] C.R.O.W., Sign up for the bike - Design manual for a cycle-friendly infrastructure, Record 10, The Netherlands C.R.O.W, 1993. 